\title{
ON TWO THEOREMS OF JESSEN
}

\author{
DOROTHY MAHARAM
}

1. Introduction. Let $X$ be the measure-theoretic product of a sequence of measure spaces $X_{1}, X_{2}, \cdots, X_{n}, \cdots$, each of measure 1 , and let $f$ be a summable ${ }^{1}$ real-valued function on $X$. Jessen ${ }^{2}$ has proved the following two theorems: As $n \rightarrow \infty$, and for almost every $x=\left(x_{1}, x_{2}, \cdots\right)$, we have

$$
\iint \cdots f(x) d x_{n} d x_{n+1} \cdots \rightarrow f(x)
$$

and

$$
\iint \cdots \int f(x) d x_{1} d x_{2} \cdots d x_{n} \rightarrow \int_{X} f(x) d x .
$$

We shall extend these theorems to products of arbitrarily many factor spaces. ${ }^{3}$ The most natural such extension would be to take the integrals on the left-hand sides to be over all finite subsets of the factor spaces for (2), and over the complementary sets of factors for (1), and interpret the limits as directed limits. This does lead to results on mean convergence; but we shall be concerned with pointwise convergence, for which Dieudonné [4] has shown this natural extension is false, at least for (1). Apparently the order of the factors is significant. We shall show that the extension is valid if the factors are well-ordered, the limits being transfinite limits. The proofs of the two extended theorems are very similar, so we give the argument only for (1); it depends on a lemma on the convergence of countable transfinite sequences of measurable functions ( $\$ 3$ ) which may be of some interest in itself.

It may be remarked that the restriction of finite integrability of $f$ is easily seen to be superfluous in (2), but is essential in (1), even if the spaces $X_{n}$ are unit intervals. (Example: $f\left(x_{1}, x_{2}, \cdots\right)=\sum 1 / 4^{n} x_{n}$.)

All measures occurring below are to be countably additive, nonnegative, $\sigma$-finite and complete; and the measure on a product space is always to be the product measure.

Received by the editors May 2, 1958

1 That is, $f$ is measurable and $\int_{X}|f(x)| d x<\infty$.

2 [3]; see also [1, pp. 23-25; $2 ; 4]$.

3 The extended form of (1) is needed in a forthcoming paper of the author. 
2. Cylinder sets and functions. Suppose that $Y$ and $Z$ are measure spaces, and that $X$ is their product $Y \times Z$. A measurable subset $A$ of $X$ is said to be a cylinder on $Y$ if it is of the form $B \times Z$ where $B$ is a (necessarily measurable) subset of $Y$. (The empty set is, of course, always a cylinder on $Y$.) Equivalently, $A$ is a cylinder on $Y$ if and only if, for each $y \in Y$, the "fibre" $y \times Z$ is either contained in or disjoint from $A$.

Similarly, a real-valued measurable function $f$, defined on a measurable subset of $X$, is a cylinder function on $Y$ if there exists a (necessarily measurable) function $g$, defined on a subset of $Y$, such that for each $y \in Y$ and $z \in Z$ we have $f(y, z)=g(y)$. (Here, and throughout this paper, an unqualified equality between functions means that if either is defined then so is the other and they are equal.) It follows that the set on which $f$ is defined is itself a cylinder on $Y$. An equivalent condition for $f$ to be a cylinder function on $Y$ is that, for each real (or rational) number $\rho$, the set $\{(y, z) \mid f(y, z)>\rho\}$ is a cylinder set on $Y$.

It will be convenient to note the following obvious facts for reference.

(3) If $f$ is a cylinder function on $Y$, and meas $Z=1$, then $f(y, z)$ $=\int_{z} f(y, z) d z$ (whenever either has a meaning).

(4) If $f$ is a summable function, defined almost everywhere on $Y \times Z \times T$, which is a cylinder function on $Y \times T$ and if meas $Z=1$, then

$$
\int_{T} f(y, z, t) d t=\iint_{z_{\times} T} f(y, z, t) d z d t
$$

and the common set on which they fail to exist is a null cylinder on $Y$.

Now let $X$ be the product of an uncountable family $\left\{X_{\lambda} \mid \lambda \in \Lambda\right\}$ of measure spaces $X_{\lambda}$, each of total measure 1. A sub-product $Y$ $=\prod\left\{X_{\lambda} \mid \lambda \in M\right\}$ (where $M$ is a nonempty proper subset of $\Lambda$ ) is said to be "countable" if $\mathrm{M}$ is countable. For any sub-product $Y$, if $Z$ denotes the product of the remaining factors $\left\{X_{\lambda} \mid \lambda \in \Lambda-M\right\}$, the product $Y \times Z$ is of course isomorphic to $X$, and we shall not distinguish between them. The properties of $X$ can to a large extent be deduced from those of countable products, in view of the following results.

(5) Given any measurable subset $A$ of $X$, there exist a countable subproduct $Y$, and cylinders $A_{1}, A_{2}$ on $Y$, such that $A_{1} \subset A \subset A_{2}$ and

$$
\text { meas }\left(A_{2}-A_{1}\right)=0 \text {. }
$$

For the family of subsets of $X$ having this property is a Borel field containing the cylinders on products of finitely many factors, and 
thus containing also all null sets. Hence it contains all measurable sets.

(6) Each measurable function $f$ on $X$ differs from a cylinder function on some countable sub-product by a null function.

This is proved by applying (5) to the sets $\{x \mid f(x)>\rho\}$, where $\rho$ runs over the rational numbers.

Further, applying (5) to the set $\{x \mid g(x) \neq 0\}$, we obtain:

(7) Each null function $g$ on $X$ vanishes outside some null set which is a cylinder on a countable sub-product.

\section{Transfinite sequences of functions.}

Lemma. Let $\alpha^{*}$ be a countable limit ordinal, and let $\left\{f_{\gamma} \mid \gamma<\alpha^{*}\right\}$ be a transfinite sequence of measurable real-valued functions on a measure space $X$, with the property that every cofinal simple sub-sequence of $\left\{f_{\gamma}\right\}$ converges for almost all $x \in X{ }^{4}$ Then there is a measurable function $f$ on $X$ such that, for almost all $x \in X, f_{\gamma}(x) \rightarrow f(x)$ as $\gamma \rightarrow \alpha^{*}$.

We first show that there is a simple cofinal sub-sequence $\left\{f_{\gamma(n)}\right\}(n=1,2, \cdots)$, with $\gamma(1)<\gamma(2)<\cdots$ and $\sup \gamma(n)=\alpha^{*}$, such that $\lim \sup f_{\gamma(n)}(x)=\lim \sup f_{\gamma}(x)$ for almost all $x$. There is no loss of generality in assuming that meas $X=1$, and that the functions $f_{\gamma}$ are uniformly bounded. Now take a sequence $\alpha_{n}(n=1,2, \cdots)$ of ordinals such that $\alpha_{1}<\alpha_{2}<\cdots$ and $\sup \alpha_{n}=\alpha^{*}$. Define $\beta_{1}=\alpha_{1}$, $g_{1}(x)=\sup \left\{f_{\gamma}(x) \mid \beta_{1} \leqq \gamma<\alpha^{*}\right\}$. By renumbering the (countably many) functions $f_{\gamma}$ occurring here into a simple sequence, it is easy to see that we can choose a finite number of values of $\gamma$, say $\gamma(1), \gamma(2), \cdots$, $\gamma\left(n_{1}\right)$, such that $\beta_{1} \leqq \gamma(i)<\alpha^{*}$ and $\max \left\{f_{\gamma(1)}, \cdots, f_{\gamma\left(n_{1}\right)}\right\}>g_{1}(x)-1$ except on a subset $E_{1}$ of $X$ of measure less than $1 / 2$. We may of course suppose that $\gamma(1)<\gamma(2)<\cdots<\gamma\left(n_{1}\right)<\alpha^{*}$. We iterate the process, taking $\beta_{2}=\max \left(\gamma\left(n_{1}\right)+1, \alpha_{2}\right)$ and $g_{2}(x)=\sup \left\{f_{\gamma}(x) \mid \beta_{2} \leqq \gamma<\alpha^{*}\right\}$; and so on. In this way we construct recursively (for $i=2,3, \cdots$ ) an increasing sequence of integers $n_{i}$ and an increasing sequence of ordinals $\gamma(1), \cdots, \gamma\left(n_{i}\right), \cdots$, so that, on writing $\beta_{i}=\max \left(\alpha_{i}, \gamma\left(n_{i-1}\right)+1\right)$ and $g_{i}(x)=\sup \left\{f_{\gamma}(x) \mid \beta_{i} \leqq \gamma<\alpha^{*}\right\}$, we have

$$
\max \left\{f_{\gamma(m)}(x) \mid n_{i-1}+1 \leqq m \leqq n_{i}\right\}>g_{i}(x)-1 / i
$$

except for $x \in E_{i}$, where meas $E_{i}<2^{-i}$. Clearly sup $\gamma(n)=\alpha^{*}$, for $\gamma\left(n_{i}\right) \geqq \alpha_{i}$. Let $k(x)=\lim \sup \left\{f_{\gamma(n)}(x) \mid n=1,2, \cdots\right\}$; it is easy to see that $k(x) \leqq \lim \sup f_{\gamma}(x)$ for each $x \in X$, and that $k(x) \geqq \lim \sup f_{\gamma}(x)$ $-1 / m$ if $x \in U\left\{E_{i} \mid i \geqq m\right\}$, a set of measure less than $1 / 2^{m-1}$. It follows that $k(x)=\lim \sup f_{\gamma}(x)$ almost everywhere.

${ }^{4}$ Note that the exceptional null set will depend, in general, on the subsequence. 
Similarly we construct a sequence of ordinals $\delta(1)<\delta(2)<\ldots$ $<\alpha^{*}$, with $\sup \delta(n)=\alpha^{*}$ and $\lim \inf f_{\delta(n)}(x)=\lim \inf f_{\gamma}(x)$ almost everywhere. The combined set of ordinals $\gamma(1), \gamma(2), \cdots, \delta(1)$, $\delta(2), \cdots$, numbered in increasing order as a single sequence, provides a cofinal simple subsequence of $\left\{f_{\gamma}\right\}$ which has almost everywhere the same lim sup and $\lim \inf$ as $\left\{f_{\gamma}\right\}$. By hypothesis, this subsequence converges almost everywhere to a (necessarily measurable) function $f$; hence $f_{\gamma}(x) \rightarrow f(x)$ almost everywhere.

4. The theorems. For any limit ordinal $\alpha^{*}$, let $\left\{X_{\alpha} \mid \alpha<\alpha^{*}\right\}$ be a well-ordered collection of measure spaces, each of measure 1 , and let $X=\prod X_{\alpha}$. For each ordinal $\gamma<\alpha^{*}$ (but $>1$ ) we write $Y_{\gamma}$ $=\prod\left\{X_{\alpha} \mid \alpha<\gamma\right\}, \quad Z_{\gamma}=\prod\left\{X_{\alpha} \mid \gamma \leqq \alpha<\alpha^{*}\right\}$, and identify $X$ with $Y_{\gamma} \times Z_{\gamma}$. Let $f$ be any integrable real-valued function on $X$ (the values $\pm \infty$ are allowed), and let $f_{\gamma}$ be the cylinder function on $Y_{\gamma}$ defined (almost everywhere) by: $f_{\gamma}(x)=f_{\gamma}\left(y_{\gamma}, z_{\gamma}\right)=\int_{z_{\gamma}} f\left(y_{\gamma}, z_{\gamma}\right) d z_{\gamma}$. Similarly we define $f^{\gamma}$ to be the cylinder function on $Z_{\gamma}$ defined (almost everywhere) by

$$
f^{\gamma}(x)=f^{\gamma}\left(y_{\gamma}, z_{\gamma}\right)=\int_{Y_{\gamma}} f\left(y_{\gamma}, z_{\gamma}\right) d y .
$$

THEOREM 1. If $\int_{X}|f(x)| d x<\infty$, then $f_{\gamma}(x) \rightarrow f(x)$ as $\gamma \rightarrow \alpha^{*}$, for almost every $x \in X$.

Theorem 2. $f^{\gamma}(x) \rightarrow \int_{X} f(x) d x$ as $\gamma \rightarrow \alpha^{*}$, for almost every $x \in X$.

The proof of Theorem 1 is built up by combining several special cases.

I. If $\alpha^{*}=\omega_{0}$, the theorem reduces to Jessen's original theorem (1).

II. Suppose now that $\alpha^{*}$ is countable, and let $\left\{\beta_{n}\right\}(n=1,2, \cdots)$ be a cofinal simple sequence (i.e., $\beta_{1}<\beta_{2}<\cdots$ and $\sup \beta_{n}=\alpha^{*}$ ). We may assume $\beta_{1}>1$. Let $R_{1}=\prod\left\{X_{\alpha} \mid \alpha<\beta_{1}\right\}$, and $R_{n}=\prod\left\{X_{\alpha} \mid \beta_{n-1}\right.$ $\left.\leqq \alpha<\beta_{n}\right\} \quad(n=2,3, \cdots)$, and write $\bar{Y}_{n}=R_{1} \times R_{2} \times \cdots \times R_{n}, \bar{Z}_{n}$ $=\prod\left\{X_{\alpha} \mid \beta_{n} \leqq \alpha<\alpha^{*}\right\}$; thus $X=\prod R_{n}=\bar{Y}_{n} \times \bar{Z}_{n}$. From I we obtain that, if

$$
\bar{f}_{n}(x)=\int_{\bar{z}_{n}} f\left(\bar{y}_{n}, \bar{z}_{n}\right) d \bar{z}_{n}
$$

then $\bar{f}_{n}(x) \rightarrow f(x)$ for almost all $x$. But $\bar{Y}_{n}=Y_{\beta_{n}}, \bar{Z}_{n}=Z_{\beta_{n}}$ and $\bar{f}_{n}=f_{\beta_{n}}$. Thus every simple cofinal sub-sequence $\left\{f_{\beta_{n}}\right\}$ of $\left\{f_{\gamma}\right\}$ converges to $f$ almost everywhere; and, by the lemma, so does $\left\{f_{\gamma}\right\}$.

III. Next, let $\alpha^{*}$ be uncountable, but suppose that $f$ is a cylinder function on a countable subproduct, say on $\prod\left\{X_{\alpha} \mid \alpha \in B\right\}$ where $B$ is countable; and let $\sup B=\alpha_{0}$. If $\alpha_{0}<\alpha^{*}$, we observe that, when- 
ever $\alpha_{0}<\gamma<\alpha^{*}, f_{\gamma}(x)=f(x)$ for all $x \in X$ (by (3), applied to $Y=Y_{\gamma}$ and $Z=Z_{\gamma}$ ), so trivially $f_{\gamma}(x) \rightarrow f(x) .{ }^{5}$ If however $\alpha_{0}=\alpha^{*}$, let $\beta(\gamma)$ denote, for each $\gamma<\alpha^{*}$, the least member of $B$ which $\geqq \gamma$. Suppose for the moment that $\gamma<\beta(\gamma)$ (i.e., $\gamma \in B$ ), and apply (4) to $Y=Y_{\gamma}$, $Z=\prod\left\{X_{\alpha} \mid \gamma \leqq \alpha<\beta(\gamma)\right\}, T=Z_{\beta(\gamma)}$; we see that $f_{\gamma}(x)=f_{\beta(\gamma)}(x)$ for all $x \in X$ (for which either is defined). ${ }^{5}$ If $\gamma=\beta(\gamma)$ this is still true, so it is enough to prove that $f_{\beta}(x) \rightarrow f(x)$ almost everywhere as $\beta \rightarrow \alpha^{*}$ through values in $B$; but this follows from II applied to the product of the spaces $\bar{X}_{\beta}=\prod\left\{X_{\alpha} \mid \beta \leqq \alpha<\beta^{+}\right\}$, where $\beta \in B$ and $\beta^{+}$denotes the successor of $\beta$ in $B$.

IV. Now suppose that $f$ is a null function. By (7), $f$ vanishes outside a null set $E$ which is a cylinder on some countable subproduct $\left\{X_{\alpha} \mid \alpha \in B\right\}$. As it is enough to prove the theorem under the assumption that $f$ is bounded, we may assume $|f(x)| \leqq M \chi(x)$ for all $x \in X$, where $M$ is a constant and $\chi$ is the characteristic function of $E$. From III there is a null set $N$ such that, if $x \in X-N$, we have $\chi_{\gamma}(x) \rightarrow \chi(x)$ as $\gamma \rightarrow \alpha^{*}$. But $\left|f_{\gamma}(x)\right| \leqq \int_{z_{\gamma}}\left|f\left(y_{\gamma}, z_{\gamma}\right)\right| d z_{\gamma} \leqq M \chi_{\gamma}(x)$, and therefore, if $x \in X-(N \cup E)$, we have $f_{\gamma}(x) \rightarrow 0=f(x)$.

Theorem 1 in full generality is now an immediate consequence of II, III and IV, in view of (6).

The proof of Theorem 2 is entirely similar; the extra argument needed to deal with the case in which $f$ is infinite on a set of positive measure, or has an infinite integral, is trivial.

REMARK. If we denote by $\{f\}$ the class of all functions which differ from $f$ by a null function, then, given $f$, there are at most countably many different classes $\left\{f_{\gamma}\right\}$ and $\left\{f^{\gamma}\right\} .^{6}$ This is shown by the reasoning in case III, together with the fact that if $f$ is null then all the functions $f_{\gamma}$ and $f^{\gamma}$ are null.

\section{REFERENCES}

1. E. S. Andersen and B. Jessen, Some limit theorems on integrals in an abstract set, Danske Vid. Selsk. Mat.-Fys. Medd. vol. 22 no. 14 (1946).

2. - Some limit theorems on set-functions, ibid. vol. 25 no. 5 (1948).

3. B. Jessen, The theory of integration in a space of an infinite number of dimensions, Acta Math. vol. 63 (1934) pp. 249-323.

4. J. Dieudonné, On a theorem of Jessen, Fund. Math. vol. 37 (1950) pp. 242-248.

5. J. L. Doob, Regularity properties of certain families of chance variables, Trans. Amer. Math. Soc. vol. 47 (1940) pp. 455-486.

\section{The University, Manchester, England}

${ }^{5}$ Because $\gamma$ runs over an uncountable set, it is important that no exceptional null set occurs here.

${ }^{6}$ However, it is easy to see that there may be uncountably many different functions $f_{\gamma}$ and $f^{\gamma}$. 